\title{
Percepción de riesgo sobre el consumo de drogas en escolares de secundaria del Perú
}

\author{
Ojeda- Oyague Nataly ${ }^{1}$, Podestá- Alegre Lorena ${ }^{1}$, Musayón-Oblitas Flor Yesenia²
}

\section{RESUMEN}

Objetivo: determinar la percepción de riesgo sobre el consumo de drogas en escolares de secundaria según perfil del escolar en las diferentes regiones del país. Material y métodos: investigación de tipo descriptiva, de corte transversal, basada en la revisión de una base de datos secundaria generada en el III Estudio Nacional de Prevención y Consumo de Drogas en escolares de secundaria, realizada por DEVIDA en las escuelas del país. La población estudiada estuvo conformada por adolescentes de 10 a 19 años. Se utilizó la base de datos proporcionada por DEVIDA y se analizó las variables a investigar por frecuencias simples y relativas. Resultados: el 67,2\% de estudiantes mujeres consideran un «gran riesgo» al consumo frecuente de marihuana, PBC, cocaína, éxtasis. Los estudiantes de 11-19 años consideran un «gran riesgo» al consumo frecuente de marihuana, PBC, cocaína, éxtasis entre 57,3 y 66,6\%. En Lima, Callao y provincias los estudiantes perciben un «gran riesgo» al consumo frecuente de drogas a un $60,5 \%$. Escolares de colegios públicos (58,9\%) consideran un «gran riesgo» al consumo frecuente ocasional de marihuana, PBC, cocaína, éxtasis. Se encontró que los escolares que viven con sus parejas consideran $40,5 \%$ a «gran riesgo» el uso frecuente de drogas ilegales. Conclusiones: las mujeres mantienen una mayor percepción de riesgo al consumo de drogas en comparación a los varones; los escolares que viven en Lima perciben como un «gran riesgo» al consumo drogas. En las instituciones públicas (58,9\%) consideran «gran riesgo» al consumo de drogas ilegales. Se demuestra que la población escolar que vive con sus padres o algún familiar muestra una mayor percepción de quienes viven con sus parejas.

Palabras clave: percepción de riesgo, consumo de drogas, perfil del escolar. Perú. (Fuente DeCs BIREME).

\section{Risk perception on drug use in secondary school of Peru}

\begin{abstract}
Objetive: To determine the risk perception of drug use in secondary school by school profile in different regions of the country. Material and methods: The research is descriptive, cross-sectional. It is based on a review of a secondary database generated in the Third National Study of Drug Abuse Prevention and in secondary school, by DEVIDA in schools in the country. The study population consisted of adolescents aged 10 to 19 years. We used the database provided by DEVIDA and analyzed the variables to investigate simple and relative frequencies. Results: The female students (67, 2\%) considered a «great risk» to the frequent consumption of marijuana, PBC, cocaine, ecstasy. Students 11-19 years considered a "great risk» to the frequent consumption of marijuana, PBC, cocaine, ecstasy between 57,3\% and 66,6\%. In Lima, Callao and Provinces students perceive «great risk» frequent drug consumption to 60,5\%. Teaching in public schools (58, 9\%) considered a «great risk» frequent occasional marijuana use, PBC, cocaine, ecstasy. It was found that the students who live with their partners consider $40,5 \%$ to «high risk» frequent use of illegal drugs. Conclusions: Women have a higher risk perception of drug use compared to boys; school children living in Lima perceive «great risk» to use drugs. In public institutions (58,9\%) considered «high risk» to illegal drug use. We show that the school population who live with their parents or a family member shows a greater perception of those living with their partners.
\end{abstract}

Key words: risk perception, drug use, school profile. Peru. (Source DeCs BIREME).

\footnotetext{
Alumnas de la Facultad de Enfermería de la Universidad Peruana Cayetano Heredia.

Doctora en Salud Pública, Profesora principal de la Universidad Peruana Cayetano Heredia.
} 


\section{INTRODUCCIÓN}

La percepción de riesgo sobre el de consumo de drogas (1) mide la sensibilidad sobre el tema de las drogas, mediante los mensajes preventivos acerca del riesgo de consumo de drogas transmitidas dentro y fuera de la institución educativa. Dichos mensajes ayudan a enseñar y/o fortalecer el conocimiento acerca del riesgo de consumo de sustancias entre adolescentes y jóvenes. Este indicador permite evaluar la influencia que ejerce el discurso preventivo en el uso de drogas de la población escolar (1).

La percepción de riesgo es un proceso cognitivo que descansa en la información de cada persona acerca de diferentes cuestiones como contextos, personas, objetos, y que procesa de forma inmediata organizando un juicio o valor; se podría añadir que ese juicio o valor condicionará su comportamiento (2). La percepción de riesgo estaría ubicada también en el plano subjetivo, dado que los factores que intervienen en su configuración serían perceptivos, de historia personal (experiencias), tomaría en cuenta la cantidad y calidad de la información, las creencias, las actitudes, los estereotipos y las motivaciones (2).

El III Estudio de Prevención y Consumo de Drogas, registró un alto incremento en el consumo de alcohol, de cigarrillos, también de pasta básica de cocaína (PBC) y cocaína, al igual que el éxtasis y la marihuana, donde el desconocimiento y/o el bajo riesgo atribuido a las drogas es un factor que toma importancia dentro de la población de escolares de 11 a 19 años, de nivel secundario (1). En las distintas regiones del país, los escolares crean percepciones de riesgos sobre el consumo de drogas según sus conocimientos y a la exposición en la que se encuentre.

Arrellanez et al. determinaron que el grado de información que recibe un joven sobre drogas está relacionado con su esfera personal, su ambiente familiar, escolar y sus grupos de amigos (3). En Lima, Salazar et al. (4) realizaron una investigación sobre las percepciones sociales sobre drogas y explicaron que la percepción predominante de la gente se ha vuelto más permisiva respecto al consumo de drogas. Pero esta percepción se acompaña de una fuerte crítica, sobre todo de parte de las mujeres, quienes perciben que no se hace nada (ni individual, ni colectivamente; ni desde los poderes públicos) y que, además, no existe repuesta ante una situación en la que el consumo es mucho más visible. El consumo de la drogas es percibido como un acto de voluntad propia y, como tal, viene o vendrá condicionado por los valores inculcados. En la medida en que estos sean lo suficientemente potentes para superar los peligros y tomar decisiones adecuadas, la pe- ligrosidad de las drogas se verá reducida (4).

El «Informe del Uso de Drogas en Las Américas» (OEA 2011), reporta que existe una percepción de gran riesgo asociado al consumo de drogas, considerado como un punto de referencia subjetivo importante para incrementar la probabilidad de uso de sustancias psicoactivas. Es posible observar la influencia de una percepción negativa acerca de las drogas en las tasas de consumo. En concreto, los países donde el riesgo percibido asociado al consumo de drogas es más elevado, tienden a tener una menor prevalencia de consumo (5). Este patrón es válido para casi cualquier droga y se ha observado también en estudios realizados en otros países. La comprensión de la percepción del riesgo asociada al consumo de drogas es un elemento esencial para la planificación y ejecución de programas de prevención de drogas (5).

Existen muchas teorías que podrían explicar la percepción de riesgo de un adolescente. El modelo de creencias de salud (MCS) se consolidó como uno de los más potentes y utilizados para estudiar la conducta de salud a partir de la predicción del comportamiento. El modelo se fundamenta en la explicación y la predicción de los comportamientos saludables que se generan en función de una serie de creencias que las personas elaboran a partir de acontecimientos relacionados con la salud. La teoría de la acción razonada y la conducta planeada, por su parte, muestra los planteamientos más clásicos de la teoría de las actitudes, el concepto de actitud se ha enmarcado en el ámbito social dado que surge de la interrelación entre el sujeto y su entorno. A partir de la década de los sesenta del pasado siglo aparecen los denominados 'modelos de componente único' afirmando que solamente existe un componente en la actitud, el que se determina como evaluativo. Desde esta orientación, las teorías de la acción razonada y la conducta planeada, tuvieron como sustento primario intentar dar una explicación empírica al comportamiento social y, posteriormente, se derivaron al estudio de la conducta de salud, siendo uno de los objetos de estudio la prevención del consumo de tabaco y alcohol (6).

En la teoría de la motivación protectora se parte de la premisa básica de que el miedo a las consecuencias mediatiza el comportamiento final de las personas, motivándolas a buscar medidas de protección (7). Según Medina y León (8), la teoría afirmó que se puede aumentar significativamente la intención de mantener una conducta de salud si se consigue infundir el suficiente miedo en el sujeto, siempre y cuando se le dé también las salidas posibles para evitar el proceso de enfermedad. Una vez que el sujeto está motivado hacia la protección los com- 
portamientos que lleve a cabo irán en consonancia con la evitación del riesgo (8).

En el país se han reportado pocas investigaciones que evalúen la percepción de riesgo sobre el consumo de drogas, la Comisión Nacional para el Desarrollo y Vida sin Drogas (DEVIDA) es una de las pocas entidades que realizan este tipo de investigaciones. Desde el campo de la enfermería, la comprensión del fenómeno de las drogas permitirá abordar programas de intervención de carácter preventivo, los cuales podrán tener un efecto mayor si se identifican los grupos vulnerables o de mayor riesgo, para el caso en mención, los adolescentes con una percepción de riesgo débil o nulo hacia el consumo de drogas.

El estudio tuvo por objetivo determinar la percepción de riesgo sobre el consumo de drogas en escolares de secundaria según perfil del escolar en las diferentes regiones del país.

\section{MATERIAL Y MÉTODOS}

Investigación descriptiva, de corte transversal. Se realizó la revisión de una base de datos secundaria generada por el III Estudio Nacional de Prevención y Consumo de Drogas, realizado por DEVIDA en el año 2009, en escolares de 11 a 19 años matriculados en secundaria. DEVIDA autorizó la realización del estudio. Este trabajo solo usó un segmento de la base de datos de la encuesta: la sección I, con datos referentes al colegio con dos preguntas, como el tipo de colegio, y el tipo de colegio por sexo (sección I, preguntas 1- 2); la sección II, con los datos de los escolares como: edad, sexo, año de estudio y con quien viven (sección II, preguntas 3 -8); finalmente, la sección VIII, donde se evaluó la percepción de riesgo en los escolares, que fue evaluada mediante 18 preguntas (sección VIII, preguntas 24 - 24.17) que abarcaron la percepción de riesgo hacia el consumo de alcohol, cigarrillos, tranquilizantes, drogas ilegales como PBC, cocaína, inhalantes (Terokal) y éxtasis; drogas médicas como tranquilizantes y estimulantes, las que fueron evaluadas con una graduación de: «no sabe» del riesgo, no percibe «ningún riesgo», percibe un «riesgo leve», percibe un «riesgo moderado» y percibe un «gran riesgo».

Se realizó el control de calidad de la base de datos recibida de DEVIDA, mediante el análisis de las frecuencias simples de cada una de las variables consignadas en la base completa, tratando de identificar codificación errónea o datos missing. Posteriormente, se realizó el cruce de las variables de la sección VIII (preguntas 24-24.17) con las de la sección I (preguntas 1-2) y sección II (pre- guntas 3-8). Los datos fueron presentados en frecuencias simples y relativas.

\section{RESULTADOS}

En la tabla 1 puede observarse que el 34,5\% de escolares varones y el 37,2\% de escolares mujeres tienen una percepción de «riesgo leve» cuando se consume alcohol alguna vez, mientras que el $56,9 \%$ y el $65,6 \%$ de los escolares varones y mujeres, respectivamente, consideran un «gran riesgo» cuando el consumo de alcohol es frecuente. Los escolares consideran que existe un gran riesgo cuando se consume alcohol hasta emborracharse (54,3\% de mujeres y $48,1 \%$ de varones).

Los estudiantes varones consideran un «gran riesgo» el consumo ocasional de marihuana, PBC, cocaína o éxtasis con un 33,1; 36,5; 35,1 y 35,8\% respectivamente. Mientras que el consumo frecuente es considerado de «gran riesgo» entre 59 y 60\% de los varones para las mismas drogas. En el caso de escolares mujeres, la percepción de que el consumo es de "gran riesgo» para el mismo tipo de drogas, si el consumo es ocasional, asciende en uno o dos puntos porcentuales a lo percibido por los varones, mientras que cuando el consumo de dichas drogas es frecuente la percepción de riesgo es entre cinco y siete puntos porcentuales más alta que lo que perciben los varones. En el caso de consumo de inhalantes el $49,9 \%$ de la población escolar de varones considera de «gran riesgo» el aspirado de inhalantes alguna vez, mientras que el 55,19\% considera de "gran riesgo» el aspirado de inhalantes de manera frecuente. En el caso de las mujeres, la percepción de que los inhalantes son de «gran riesgo» cuando el consumo es alguna vez es tres puntos porcentuales más que los varones y se duplica cuando consideran que el consumo es frecuente.

En cuanto a las drogas médicas, el 52,53\% de los escolares varones consideran de gran riesgo el consumo de drogas médicas de manera frecuente; en cuanto al consumo ocasional el 39,5\%.de los escolares varones consideran el consumo de «gran riesgo». Esta percepción se incrementa en las mujeres, en el caso del consumo ocasional, hasta el 43,5\%, dicha percepción sube 50\% más cuando el consumo es frecuente.

En la tabla 2 puede observarse que el 35,8\% de los escolares encuestados entre las edades de 17 a 19 años y 37,8\% entre los 14 y 16 años, tienen una percepción de «riesgo leve» del consumo ocasional de alcohol, mientras que entre $62,4 \%$ y el $63,4 \%$ de los escolares de las respectivas edades consideran un «gran riesgo» el consumo 
frecuente de alcohol, y entre un 51,7 y 52,4\% de las edades correspondientes consideran que el «gran riesgo» es consumir alcohol hasta emborracharse. En el caso de cigarrillos, entre un 34,7\% de escolares entre 17 y 19 años y un $37,1 \%$ entre 14 y 16 años, considera de «riesgo leve» el consumo ocasional, mientras que el consumo frecuente es considerado de "gran riesgo» para el 60,4\% y el 61,1\% de los escolares de los mismos grupos etarios, respectivamente.

Los estudiantes de 11 a 13 años de edad, consideran un «gran riesgo» el consumo ocasional de marihuana, PBC, cocaína, éxtasis entre el 33,4; 35,3; 34,4 y el 34,6\% respectivamente, mientras que la percepción de «gran riesgo» para escolares de la misma edad oscila entre el 57 y $60 \%$ cuando el consumo es frecuente. En el caso de consumo de inhalantes, el 52,9\% de la población escolar de 14 a 16 años considera de "gran riesgo» el aspirado de inhalantes alguna vez, mientras que el $61,2 \%$ de jóvenes de la misma edad considera que el aspirado de inhalantes frecuentemente es de «gran riesgo».

El consumo de las drogas médicas, como los tranquilizantes es considerada de "gran riesgo", cuando el consumo es frecuente, por los escolares de edades de 14 a16 y de 17 a 19 años con un 59,2\% y un 58,8\% respectivamente; en cuanto al consumo ocasional el $41,6 \%$ y el $44,8 \%$ de las mismas edades considera que el consumo es de «gran riesgo».

De acuerdo a la tabla 3, el 35,0\% de escolares de colegios en provincias, el 37,0\% de escolares de colegios de la provincia constitucional del Callao y el 41,8 \% de escolares de colegios en Lima, consideran de «riesgo leve» el haber consumido alcohol alguna vez, mientras que entre $58,9 \%$ de escolares de colegios del Callao y el $63,5 \%$ de los escolares de colegios de Lima consideran un «gran riesgo» cuando el consumo de alcohol es frecuente y el $50,2 \%$ de escolares de colegios de Provincia y el 58,5 \% de escolares de colegios de Lima consideran un «gran riesgo» cuando el consumo de alcohol es hasta emborracharse. Por su parte, el 34,1\% de escolares de colegios de provincia y un $42,2 \%$ de escolares de colegios de Lima consideran de «riesgo leve» el consumo de alguna vez de cigarrillos, mientras que el 56,8\% de escolares de colegios del Callao y el 62,1\% de escolares de colegios de Lima consideran un «gran riesgo» el consumo de cigarrillos de manera frecuente.

Entre el 32,3 y el 37,7\% de los estudiantes de escuelas del Callao perciben un «gran riesgo» el consumo ocasional de marihuana, PBC, cocaína y éxtasis, mientras que si su consumo es frecuente la percepción de «gran riesgo» casi se duplica entre un 65,3 y 68,2\%, para los escolares de las mismas escuelas. Las drogas médicas, como los tranquilizantes, se consideran de «gran riesgo» por 56,3 al 63,1\% de los escolares en las diferentes ubicaciones geográficas cuando el consumo es frecuente, pero cuando el consumo es ocasional o de alguna vez, el porcentaje solo entre 41,3 y $42,4 \%$ de los escolares del mismo perfil lo consideran de «gran riesgo».

Un 34,4\% de escolares de colegios públicos y un 41.1\% que estudian en colegios privados perciben un «riesgo leve» al consumo de alcohol alguna vez, mientras que entre un $59,7 \%$ de escolares de colegios públicos y un $66,3 \%$ de colegios privados consideran un «gran riesgo» el consumo frecuente de alcohol y el 59,6\% de escolares de colegios privados perciben de «gran riesgo» el consumo de alcohol hasta emborracharse según puede verse en la tabla 4. En el caso del consumo de cigarrillos, el 33,6\% de escolares de colegios públicos y el $41,0 \%$ de escolares de escuelas privadas considera un «riesgo leve» el consumo ocasional, y sobre el consumo frecuente, es considerado por el $65,1 \%$ de escolares de colegios privados como de «gran riesgo».

Entre el 32,6 y el 55,9\% de escolares de colegios públicos y privados respectivamente, consideran un "gran riesgo» el consumo ocasional de marihuana, PBC, cocaína, éxtasis; al consumo frecuente lo consideran de "gran riesgo» entre 58,9 y $74,5 \%$ de los escolares con el mismo perfil. En el caso de consumo de inhalantes, un 55,9\% de escolares de colegios privados considera de «gran riesgo» el aspirado de inhalantes alguna vez, mientras que el 69,2\% considera de «gran riesgo» el aspirado de inhalantes de manera frecuente. Un 40,7\% des escolares señalaron de «gran riesgo» el consumo ocasional, y un $64,3 \%$ al consumo frecuente de drogas médicas.

En la tabla 5 se observa que el $35,8 \%$ de escolares que viven con su padre y madre perciben como un «riesgo leve» al consumo de alcohol alguna vez, mientras que $61,4 \%$ que presenta el mismo perfil familiar consideran un «gran riesgo» el consumo frecuente de alcohol. En el caso de cigarrillos el 35,0\% de escolares que viven con su padre y madre perciben como un «riesgo leve» al consumo alguna vez, y sobre el consumo frecuente es considerado por el 59,6\% de escolares con el mismo perfil familiar como un «gran riesgo».

Entre el 30,0 y 32,4\% de la población escolar que vive con su pareja o conviviente percibe de «gran riesgo» al consumo ocasional de marihuana, PBC, cocaína y éxtasis, y al 
Tabla 1. Percepción de riesgo del consumo ocasional y/o frecuente de drogas en la población escolar de secundaria según sexo a nivel nacional

\begin{tabular}{|c|c|c|c|c|c|c|c|}
\hline \multirow[b]{2}{*}{ Tipo de consumo } & \multirow[b]{2}{*}{ Sexo } & \multicolumn{6}{|c|}{ Percepción de riesgo según sexo } \\
\hline & & $\begin{array}{c}\text { Ningún } \\
\text { riesgo }\end{array}$ & Riesgo leve & $\begin{array}{c}\text { Riesgo } \\
\text { moderado }\end{array}$ & $\begin{array}{c}\text { Gran } \\
\text { riesgo }\end{array}$ & No sabe & $\begin{array}{c}\text { No } \\
\text { responde }\end{array}$ \\
\hline \multicolumn{8}{|c|}{ Drogas legales } \\
\hline \multicolumn{8}{|l|}{ Alcohol } \\
\hline \multirow{2}{*}{ Alguna vez } & $\mathrm{F}$ & 13,6 & 37,2 & 19,7 & 15,6 & 9,7 & 4,2 \\
\hline & $\mathrm{M}$ & 15,4 & 34,5 & 18,6 & 16,1 & 11,1 & 4,3 \\
\hline \multirow{2}{*}{ Frecuentemente } & $\mathrm{F}$ & 3,4 & 6,6 & 12,6 & 65,6 & 9,6 & 2,2 \\
\hline & M & 5,3 & 7,9 & 15,4 & 56,9 & 11,9 & 2,7 \\
\hline \multirow{2}{*}{ Hasta emborracharme } & $\mathrm{F}$ & 3,9 & 10,8 & 15,7 & 54,3 & 12,8 & 2,5 \\
\hline & M & 6,1 & 11 & 17,5 & 48,1 & 14,3 & 3 \\
\hline \multicolumn{8}{|l|}{ Cigarrillos } \\
\hline \multirow{2}{*}{ Alguna vez } & $\mathrm{F}$ & 12,5 & 36,8 & 15,1 & 20,4 & 13,5 & 1,7 \\
\hline & M & 15,1 & 33,6 & 14,3 & 21,4 & 13,6 & 1,9 \\
\hline \multirow{2}{*}{ Frecuentemente } & $\mathrm{F}$ & 3,4 & 8,1 & 13,9 & 63,4 & 8,9 & 2,3 \\
\hline & M & 5,2 & 9,6 & 16,3 & 54,9 & 11,1 & 2,8 \\
\hline \multicolumn{8}{|c|}{ Drogas ilegales } \\
\hline \multicolumn{8}{|l|}{ Marihuana } \\
\hline Alguna vez & F & $\begin{array}{c}5 \\
75\end{array}$ & $\begin{array}{l}18,6 \\
179\end{array}$ & $\begin{array}{l}24,5 \\
229\end{array}$ & $\begin{array}{l}34,8 \\
331\end{array}$ & $\begin{array}{l}13,7 \\
147\end{array}$ & $\begin{array}{l}3,5 \\
39\end{array}$ \\
\hline \multirow{2}{*}{ Frecuentemente } & $\mathrm{F}$ & 3,1 & 4,4 & 8,2 & 67,2 & 13,6 & 3,5 \\
\hline & M & 4,9 & 5,3 & 9,7 & 60,58 & 15,4 & 4,2 \\
\hline \multicolumn{8}{|l|}{ Pbc o crack } \\
\hline \multirow{2}{*}{ Alguna vez } & $\mathrm{F}$ & 3,9 & 13,4 & 22,7 & 37,7 & 18,3 & 4 \\
\hline & M & 5,8 & 13,5 & 22,6 & 36,5 & 17,1 & 4,5 \\
\hline \multirow{2}{*}{ Frecuentemente } & $\mathrm{F}$ & 3,1 & 3,6 & 6,2 & 64,4 & 18,7 & 3,9 \\
\hline & M & 4,7 & 4,7 & 8,1 & 59,5 & 18,6 & 4,4 \\
\hline \multicolumn{8}{|l|}{ Cocaína } \\
\hline \multirow{2}{*}{ Alguna vez } & $\mathrm{F}$ & 4,2 & 16,2 & 25,2 & 36 & 14,4 & 3,9 \\
\hline & M & 6,1 & 15,5 & 23,8 & 35,1 & 15 & 4,5 \\
\hline \multirow{2}{*}{ Frecuentemente } & $\mathrm{F}$ & 3,2 & 3,9 & 7,4 & 67 & 14,8 & 3,7 \\
\hline & M & 4,8 & 5 & 8,9 & 60,51 & 16,4 & 4,4 \\
\hline \multicolumn{8}{|l|}{ Inhalantes } \\
\hline Alguna vez & $\mathrm{F}$ & 3,7 & 8,9 & 15,7 & 52,6 & 16,2 & 2,9 \\
\hline & M & 5,4 & 8,9 & 15,5 & 49,9 & 17,4 & 3,4 \\
\hline Frecuentemente & $\mathrm{F}$ & 3,5 & 5,6 & 8,7 & 61,9 & 17,5 & 2,8 \\
\hline & M & 5,5 & 6,7 & 10,9 & 55,19 & 18,52 & 3,3 \\
\hline Extasis & & & & & & & \\
\hline Alguna vez & $\mathrm{F}$ & 3,9 & 13,3 & 22,1 & 37,7 & 18,9 & 4,1 \\
\hline Higund veL & M & 5,7 & 13,5 & 22,3 & 35,8 & 18,2 & 4,6 \\
\hline Frecuentemente & $\mathrm{F}$ & 3,3 & 2,6 & 4,4 & 65,5 & 20,4 & 3,9 \\
\hline rrecuentemente & M & 5,2 & 3,6 & 6,8 & 59,12 & 21 & 4,3 \\
\hline & & & Drogas me & licas & & & \\
\hline Tranquilizante & & & & & & & \\
\hline Alguna vez & $\mathrm{F}$ & 5,1 & 14,9 & 19,3 & 43,5 & 14,7 & 2,5 \\
\hline & M & 7,1 & 14,5 & 19 & 39,5 & 16,8 & 3,1 \\
\hline Frecuentemente & $\mathrm{F}$ & 3,4 & 6,3 & 10,3 & 61,9 & 15,5 & 2,7 \\
\hline 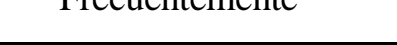 & M & 5,6 & 7,6 & 13 & 52,53 & 17,93 & 3,3 \\
\hline
\end{tabular}


Tabla 2. Percepción del riesgo de consumo ocasional y/o frecuente de drogas en la población escolar de secundaria según edad a nivel nacional

\begin{tabular}{|c|c|c|c|c|c|c|c|}
\hline \multirow[b]{2}{*}{ Tipo de consumo } & \multirow[b]{2}{*}{ Edad } & \multicolumn{6}{|c|}{ Percepción de riesgo según edad } \\
\hline & & $\begin{array}{c}\text { Ningún } \\
\text { riesgo }\end{array}$ & $\begin{array}{c}\text { Riesgo } \\
\text { leve }\end{array}$ & $\begin{array}{c}\text { Riesgo } \\
\text { moderado }\end{array}$ & $\begin{array}{c}\text { Gran } \\
\text { riesgo }\end{array}$ & No sabe & $\begin{array}{c}\text { No } \\
\text { responde } \\
\end{array}$ \\
\hline \multicolumn{8}{|c|}{ Drogas legales } \\
\hline \multirow{3}{*}{ Alguna vez } & $11-13$ & 14,2 & 32,9 & 17,7 & 17,7 & 12,7 & 4,8 \\
\hline & $14-16$ & 14,6 & 37,8 & 20,1 & 14,6 & 9,2 & 3,8 \\
\hline & $17-19$ & 14,5 & 35,8 & 19 & 17 & 9,8 & 3,9 \\
\hline \multirow{4}{*}{ Frecuentemente } & $11-13$ & 5,1 & 7,3 & 13,6 & 57,8 & 13,1 & 3,1 \\
\hline & $14-16$ & 3,7 & 7,1 & 14,4 & 63,4 & 9,4 & 2,1 \\
\hline & $17-19$ & 4,2 & 7,3 & 13,8 & 62,4 & 10,4 & 1,9 \\
\hline & $11-13$ & 5,7 & 10,6 & 15 & 49,5 & 15,7 & 3,4 \\
\hline \multirow[t]{2}{*}{ Hasta emborracharme } & $14-16$ & 4,3 & 11 & 17,6 & 52,4 & 12,5 & 2,2 \\
\hline & $17-19$ & 5,3 & 11,4 & 16,8 & 51,7 & 12,3 & 2,5 \\
\hline \multicolumn{8}{|l|}{ Cigarrillos } \\
\hline \multirow{3}{*}{ Alguna vez } & $11-13$ & 13,9 & 32,5 & 13,3 & 22 & 16 & 2,4 \\
\hline & $14-16$ & 13,8 & 37,1 & 15,6 & 19,8 & 12,2 & 1,4 \\
\hline & $17-19$ & 13,3 & 34,7 & 15,1 & 22,7 & 12,7 & 1,6 \\
\hline \multirow{3}{*}{ Frecuentemente } & $11-13$ & 5,2 & 8,7 & 14,7 & 56,1 & 12,1 & 3,2 \\
\hline & $14-16$ & 3,6 & 8,9 & 15,7 & 61,1 & 8,7 & 2,1 \\
\hline & $17-19$ & 4,1 & 9,2 & 14,5 & 60,4 & 9,8 & 2 \\
\hline \multicolumn{8}{|c|}{ Drogas ilegales } \\
\hline \multicolumn{8}{|l|}{ Marihuana } \\
\hline \multirow{3}{*}{ Alguna vez } & $11-13$ & 7 & 16,9 & 22,3 & 33,4 & 16,1 & 4,5 \\
\hline & $14-16$ & 5,7 & 19,1 & 25,1 & 34,2 & 12,9 & 3,1 \\
\hline & $17-19$ & 6,1 & 18,4 & 21,9 & 35 & 15 & 3,6 \\
\hline \multirow{3}{*}{ Frecuentemente } & $11-13$ & 4,8 & 5,2 & 8,9 & 60,2 & 16,3 & 4,6 \\
\hline & $14-16$ & 3,4 & 4,6 & 9,2 & 66,3 & 13,4 & 3,2 \\
\hline & $17-19$ & 4 & 4,7 & 8,1 & 65,1 & 14,5 & 3,6 \\
\hline \multicolumn{8}{|l|}{ Pbc o crack } \\
\hline \multirow{3}{*}{ Alguna vez } & $11-13$ & 5,7 & 13,2 & 20,9 & 35,3 & 19,9 & 5 \\
\hline & $14-16$ & 4,2 & 13,6 & 24,3 & 37,9 & 16,4 & 3,6 \\
\hline & $17-19$ & 4,7 & 13,2 & 20,5 & 39,5 & 18,1 & 4 \\
\hline \multirow{3}{*}{ Frecuentemente } & $11-13$ & 4,8 & 4,7 & 7,4 & 57,5 & 20,6 & 5 \\
\hline & $14-16$ & 3,3 & 3,8 & 7,2 & 64,8 & 17,4 & 3,5 \\
\hline & $17-19$ & 3,8 & 4,1 & 6,3 & 63 & 18,9 & 3,8 \\
\hline \multicolumn{8}{|l|}{ Cocaína } \\
\hline \multirow{3}{*}{ Alguna vez } & $11-13$ & 5,8 & 15,4 & 23 & 34,4 & 16,5 & 4,9 \\
\hline & $14-16$ & 4,6 & 16,3 & 26 & 36,1 & 13,4 & 3,6 \\
\hline & $17-19$ & 4,8 & 15,4 & 23 & 37,2 & 15,8 & 3,8 \\
\hline \multirow{3}{*}{ Frecuentemente } & $11-13$ & 4,8 & 4,8 & 8,4 & 59,8 & 17,4 & 4,7 \\
\hline & $14-16$ & 3,4 & 4,3 & 8 & 66,6 & 14,4 & 3,4 \\
\hline & $17-19$ & 3,8 & 4,2 & 8 & 64,2 & 16,1 & 3,8 \\
\hline Inhalantes & & & & & & & \\
\hline & $11-13$ & 5,3 & 9 & 15,1 & 48,1 & 18,6 & 3,9 \\
\hline Alguna vez & $14-16$ & 3,9 & 8,9 & 16,3 & 52,9 & 15,2 & 2,7 \\
\hline & $17-19$ & 4,5 & 7,8 & 14,2 & 52,9 & 17,9 & 2,7 \\
\hline & $11-13$ & 5,3 & 6,5 & 10,2 & 54,3 & 19,8 & 3,9 \\
\hline Frecuentemente & $14-16$ & 4 & 5,7 & 9,8 & 61,2 & 16,9 & 2,4 \\
\hline & $17-19$ & 4,3 & 5,7 & 8,9 & 60 & 18,6 & 2,6 \\
\hline
\end{tabular}




\begin{tabular}{|c|c|c|c|c|c|c|c|}
\hline \multicolumn{8}{|l|}{ Éxtasis } \\
\hline \multirow{3}{*}{ Alguna vez } & $11-13$ & 5,8 & 13,1 & 20,6 & 34,6 & 20,8 & 5,1 \\
\hline & $14-16$ & 4,2 & 13,7 & 23,6 & 37,7 & 17,2 & 3,7 \\
\hline & $17-19$ & 4,7 & 12,8 & 21,2 & 39,2 & 18,3 & 3,8 \\
\hline \multirow{3}{*}{ Frecuentemente } & $11-13$ & 5,3 & 3,8 & 5,8 & 57,3 & 23,1 & 4,8 \\
\hline & $14-16$ & 3,6 & 2,7 & 5,5 & 65,4 & 19,3 & 3,5 \\
\hline & $17-19$ & 4,0 & 2,8 & 4,9 & 64,2 & 20,5 & 3,6 \\
\hline \multicolumn{8}{|c|}{ Drogas médicas } \\
\hline \multicolumn{8}{|l|}{ Tranquilizante } \\
\hline \multirow{3}{*}{ Alguna vez } & $11-13$ & 7,1 & 13,9 & 17,9 & 40,1 & 17,1 & 3,5 \\
\hline & $14-16$ & 5,5 & 15,4 & 20,3 & 41,6 & 14,9 & 2,3 \\
\hline & $17-19$ & 5,5 & 13,5 & 17,5 & 44,8 & 16,2 & 2,5 \\
\hline \multirow{3}{*}{ Frecuentemente } & $11-13$ & 5,6 & 7,1 & 11,4 & 53,9 & 18,3 & 3,8 \\
\hline & $14-16$ & 3,7 & 6,7 & 12,2 & 59,2 & 15,7 & 2,5 \\
\hline & $17-19$ & 4,4 & 6,6 & 10,4 & 58,8 & 17,2 & 2,6 \\
\hline
\end{tabular}

Tabla 3. Percepción del riesgo de consumo ocasional y/o frecuente de drogas en la población escolar de secundaria según ubicación geográfica a nivel nacional

\begin{tabular}{|c|c|c|c|c|c|c|c|}
\hline \multirow[b]{2}{*}{ Tipo de consumo } & \multirow[b]{2}{*}{ Ubicación } & \multicolumn{6}{|c|}{ Percepción de riesgo según ubicación geográfica } \\
\hline & & $\begin{array}{c}\text { Ningún } \\
\text { riesgo }\end{array}$ & $\begin{array}{c}\text { Riesgo } \\
\text { leve }\end{array}$ & $\begin{array}{c}\begin{array}{c}\text { Riesgo } \\
\text { moderado }\end{array} \\
\end{array}$ & $\begin{array}{c}\text { Gran } \\
\text { riesgo }\end{array}$ & No sabe & $\begin{array}{c}\text { No } \\
\text { responde }\end{array}$ \\
\hline \multicolumn{8}{|c|}{ Drogas legales } \\
\hline \multicolumn{8}{|l|}{ Alcohol } \\
\hline \multirow{3}{*}{ Alguna vez } & Lima & 16,1 & 41,8 & 21,2 & 11,7 & 6,2 & 3,0 \\
\hline & Callao & 18,5 & 37,0 & 19,7 & 10,7 & 7,1 & 7,1 \\
\hline & Provincia & 14,2 & 35,0 & 18,9 & 16,6 & 11,1 & 4,4 \\
\hline \multirow{3}{*}{ Frecuentemente } & Lima & 2,8 & 8,0 & 17,9 & 63,5 & 6,6 & 1,1 \\
\hline & Callao & 3,3 & 8,0 & 17,5 & 58,9 & 7,1 & 5,2 \\
\hline & Provincia & 4,6 & 7,1 & 13,3 & 60,9 & 11,4 & 2,6 \\
\hline \multirow{3}{*}{$\begin{array}{l}\text { Hasta } \\
\text { emborracharme }\end{array}$} & Lima & 3,3 & 9,5 & 18,4 & 58,5 & 9,1 & 1,1 \\
\hline & Callao & 3,8 & 11,9 & 18,0 & 51,6 & 9,3 & 5,4 \\
\hline & Provincia & 5,3 & 11,1 & 16,3 & 50,2 & 14,3 & 2,9 \\
\hline \multicolumn{8}{|l|}{ Cigarrillos } \\
\hline \multirow{3}{*}{ Alguna vez } & Lima & 13,9 & 42,2 & 17,3 & 17,6 & 8,5 & 0,5 \\
\hline & Callao & 15,2 & 39,1 & 16,3 & 16,2 & 8,9 & 4,3 \\
\hline & Provincia & 13,8 & 34,1 & 14,3 & 21,5 & 14,4 & 1,9 \\
\hline \multirow{3}{*}{ Frecuentemente } & Lima & 2,8 & 9,2 & 19,1 & 62,1 & 5,7 & 1,1 \\
\hline & Callao & 3,1 & 9,8 & 18,9 & 56,8 & 5,6 & 5,8 \\
\hline & Provincia & 4,6 & 8,8 & 14,5 & 58,7 & 10,7 & 2,7 \\
\hline \multicolumn{8}{|c|}{ Drogas ilegales } \\
\hline \multicolumn{8}{|l|}{ Marihuana } \\
\hline \multirow{3}{*}{ Alguna vez } & Lima & 4,6 & 19,4 & 29,1 & 36,9 & 8,5 & 1,6 \\
\hline & Callao & 4,8 & 20,8 & 26,4 & 32,3 & 8,5 & 7,3 \\
\hline & Provincia & 6,5 & 18,0 & 22,9 & 33,6 & 15,2 & 3,9 \\
\hline \multirow{3}{*}{ Frecuentemente } & Lima & 2,4 & 4,7 & 9,7 & 72,4 & 9,1 & 1,7 \\
\hline & Callao & 2,3 & 3,8 & 10,5 & 67,8 & 8,5 & 7,1 \\
\hline & Provincia & 4,3 & 4,9 & 8,8 & 62,6 & 15,5 & 4,1 \\
\hline
\end{tabular}




\begin{tabular}{|c|c|c|c|c|c|c|c|}
\hline \multicolumn{8}{|l|}{ Pbc o crack } \\
\hline \multirow{3}{*}{ Alguna vez } & Lima & 3,4 & 13,4 & 27,6 & 42,2 & 11,5 & 1,9 \\
\hline & Callao & 3,1 & 13,2 & 25,4 & 37,7 & 12,6 & 8,0 \\
\hline & Provincia & 5,1 & 13,4 & 21,9 & 36,4 & 18,8 & 4,5 \\
\hline \multirow{3}{*}{ Frecuentemente } & Lima & 2,4 & 3,6 & 7,7 & 71,4 & 13,0 & 1,9 \\
\hline & Callao & 2,4 & 3,2 & 6,7 & 65,9 & 14,0 & 7,7 \\
\hline & Provincia & 4,2 & 4,3 & 7,1 & 60,5 & 19,6 & 4,4 \\
\hline \multicolumn{8}{|l|}{ Cocaína } \\
\hline \multirow{4}{*}{ Alguna vez } & Lima & 3,6 & 16,4 & 29,7 & 39,4 & 9,0 & 1,9 \\
\hline & Callao & 3,1 & 16,8 & 27,7 & 34,9 & 9,9 & 7,7 \\
\hline & Provincia & 5,4 & 15,8 & 23,7 & 35,0 & 15,7 & 4,5 \\
\hline & Lima & 2,5 & 4,2 & 8,7 & 72,9 & 9,9 & 1,8 \\
\hline \multirow{2}{*}{ Frecuentemente } & Callao & 2,3 & 3,5 & 9,1 & 68,2 & 9,8 & 7,1 \\
\hline & Provincia & 4,3 & 4,6 & 8,0 & 62,3 & 16,6 & 4,3 \\
\hline \multicolumn{8}{|l|}{ Inhalantes } \\
\hline \multirow{3}{*}{ Alguna vez } & Lima & 3,3 & 8,8 & 19,0 & 56,4 & 11,0 & 1,5 \\
\hline & Callao & 3,8 & 8,2 & 16,6 & 54,7 & 10,9 & 5,9 \\
\hline & Provincia & 4,7 & 8,9 & 15,1 & 50,4 & 17,6 & 3,4 \\
\hline \multirow{3}{*}{ Frecuentemente } & Lima & 2,7 & 5,9 & 11,8 & 66,3 & 12,2 & 1,2 \\
\hline & Callao & 3,4 & 5,3 & 12,0 & 60,3 & 12,9 & 6,1 \\
\hline & Provincia & 4,8 & 6,2 & 9,5 & 57,4 & 19,0 & 3,2 \\
\hline \multicolumn{8}{|c|}{ Drogas médicas } \\
\hline \multicolumn{8}{|l|}{ Tranquilizante } \\
\hline \multirow{3}{*}{ Alguna vez } & Lima & 5,0 & 16,7 & 23,8 & 42,4 & 11,0 & 1,1 \\
\hline & Callao & 5,4 & 15,0 & 21,1 & 41,8 & 11,1 & 5,6 \\
\hline & Provincia & 6,3 & 14,4 & 18,4 & 41,3 & 16,5 & 3,0 \\
\hline \multirow{3}{*}{ Frecuentemente } & Lima & 3,0 & 6,8 & 13,7 & 63,1 & 12,1 & 1,3 \\
\hline & Callao & 3,7 & 6,4 & 14,3 & 57,4 & 12,7 & 5,6 \\
\hline & Provincia & 4,7 & 7,0 & 11,3 & 56,3 & 17,5 & 3,2 \\
\hline
\end{tabular}

Tabla 4. Percepción de riesgo del consumo ocasional y/o frecuente de drogas en la población escolar de secundaria según tipo de colegio a nivel nacional

\begin{tabular}{|c|c|c|c|c|c|c|c|}
\hline \multirow[b]{2}{*}{ Tipo de consumo } & \multirow[b]{2}{*}{ Tipo de familia } & \multicolumn{6}{|c|}{ Percepción de riesgo según estabilidad familiar } \\
\hline & & $\begin{array}{c}\text { Ningún } \\
\text { riesgo }\end{array}$ & $\begin{array}{c}\text { Riesgo } \\
\text { leve }\end{array}$ & $\begin{array}{c}\text { Riesgo } \\
\text { moderado }\end{array}$ & $\begin{array}{l}\text { Gran } \\
\text { riesgo }\end{array}$ & No sabe & $\begin{array}{c}\text { No } \\
\text { responde }\end{array}$ \\
\hline \multicolumn{8}{|c|}{ Drogas legales } \\
\hline \multirow{3}{*}{ Alcohol } & & & & & & & \\
\hline & Padre y madre & 14,3 & 35,8 & 19,0 & 16,1 & 10,6 & 4,1 \\
\hline & Padre o madre & 15,4 & 36,2 & 19,5 & 14,9 & 9,6 & 4,3 \\
\hline \multirow[t]{5}{*}{ Alguna vez } & $\begin{array}{c}\text { Solo uno de los padres } \\
\text { y su pareja }\end{array}$ & 15,4 & 36,8 & 19,2 & 14,5 & 9,7 & 4,4 \\
\hline & Ninguno de los padres & 13,4 & 35,4 & 19,3 & 16,8 & 10,7 & 4,5 \\
\hline & Su pareja o conviviente & 15,0 & 23,7 & 19,9 & 18,1 & 17,8 & 5,6 \\
\hline & Padre y madre & 4,3 & 7,1 & 13,8 & 61,4 & 10,9 & 2,4 \\
\hline & Padre o madre & 4,7 & 7,4 & 14,2 & 61,4 & 9,8 & 2,5 \\
\hline \multirow[t]{5}{*}{ Frecuentemente } & $\begin{array}{c}\text { Solo uno de los padres } \\
\text { y su pareja }\end{array}$ & 3,6 & 7,4 & 15,5 & 60,7 & 10,4 & 2,4 \\
\hline & Ninguno de los padres & 3,9 & 7,9 & 14,2 & 60,5 & 11,0 & 2,5 \\
\hline & Su pareja o conviviente & 10,5 & 8,7 & 10,8 & 45,3 & 19,5 & 5,2 \\
\hline & Padre y madre & 5 & 11 & 16,7 & 51,1 & 13,7 & 2,6 \\
\hline & Padre o madre & 5,2 & 10,5 & 16,4 & 52,3 & 12,8 & 2,8 \\
\hline \multirow[t]{3}{*}{$\begin{array}{l}\text { Hasta } \\
\text { emborracharme }\end{array}$} & $\begin{array}{c}\text { Solo uno de los padres } \\
\text { y su pareja }\end{array}$ & 4,6 & 11,2 & 16,0 & 52,6 & 13,0 & 2,8 \\
\hline & Ninguno de los padres & 4,7 & 10,9 & 17,1 & 50,0 & 14,0 & 3,3 \\
\hline & Su pareja o conviviente & 11,9 & 12,2 & 16,7 & 34,2 & 20,2 & 4,9 \\
\hline
\end{tabular}




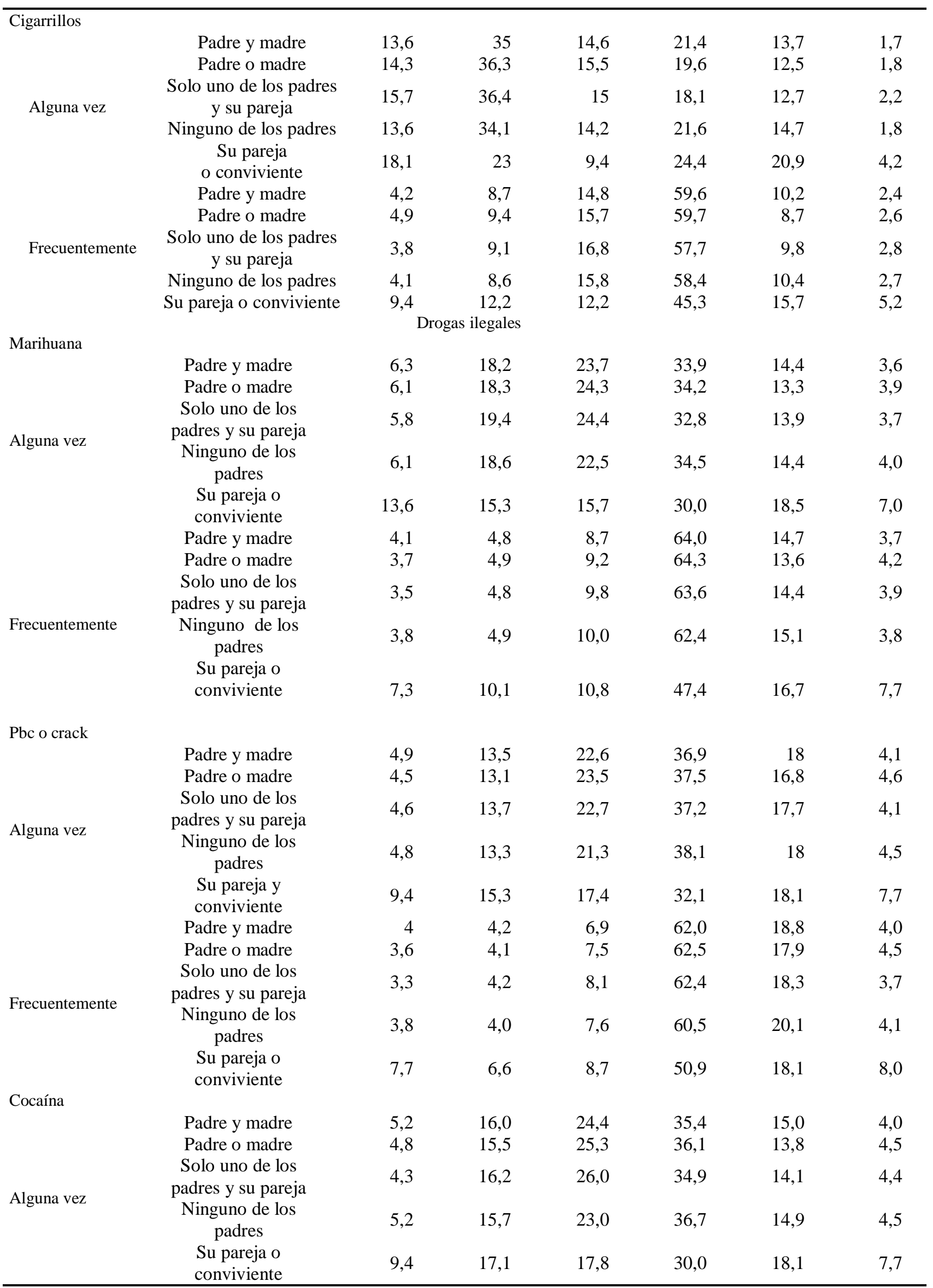




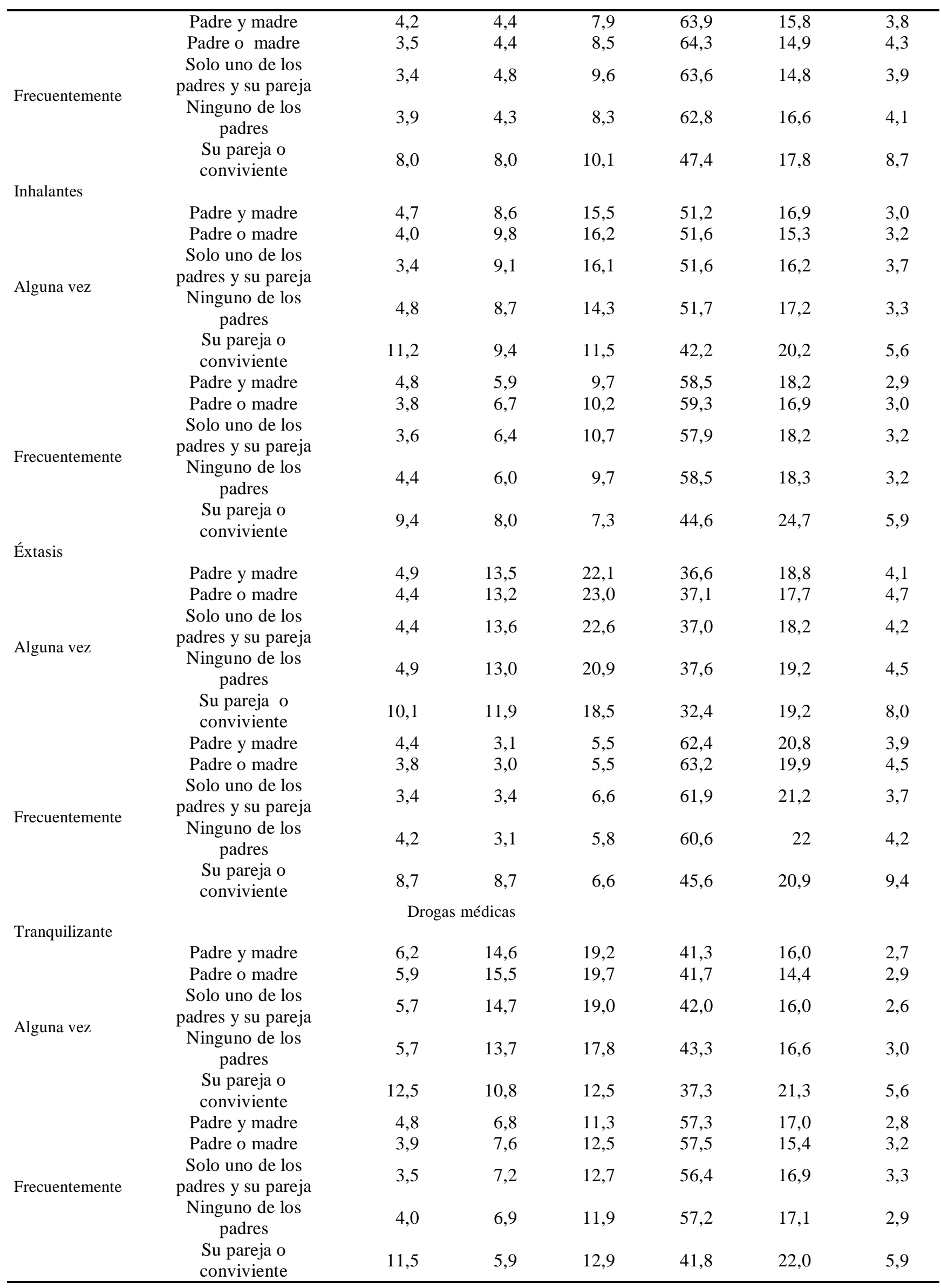


Tabla 5. PPercepción de riesgo del consumo ocasional y/o frecuente de drogas en la población escolar de secundaria según tipo de familia a nivel nacional

\begin{tabular}{|c|c|c|c|c|c|c|c|}
\hline \multirow[b]{2}{*}{ Tipo de consumo } & \multirow{2}{*}{$\begin{array}{l}\text { Tipo de } \\
\text { colegio }\end{array}$} & \multicolumn{6}{|c|}{ Percepción de riesgo según año de estudio } \\
\hline & & $\begin{array}{l}\text { Ningún } \\
\text { riesgo }\end{array}$ & $\begin{array}{l}\text { Riesgo } \\
\text { leve }\end{array}$ & $\begin{array}{c}\text { Riesgo } \\
\text { moderado }\end{array}$ & $\begin{array}{c}\text { Gran } \\
\text { riesgo }\end{array}$ & No sabe & $\begin{array}{c}\text { No } \\
\text { responde }\end{array}$ \\
\hline \multicolumn{8}{|c|}{ Drogas legales } \\
\hline \multicolumn{8}{|l|}{ Alcohol } \\
\hline \multirow{2}{*}{ Alguna vez } & Público & 14,0 & 34,4 & 18,5 & 16,8 & 11,6 & 4,8 \\
\hline & Privado & 16,5 & 41,1 & 21,5 & 12,1 & 6,0 & 2,9 \\
\hline \multirow{2}{*}{ Frecuentemente } & Publico & 4,9 & 7,5 & 13,2 & 59,7 & 11,9 & 2,8 \\
\hline & Privado & 2,4 & 6,4 & 16,9 & 66,3 & 6,5 & 1,4 \\
\hline \multirow{2}{*}{ Hasta emborracharme } & Público & 5,5 & 11,4 & 16,3 & 48,9 & 14,8 & 3,1 \\
\hline & Privado & 3,0 & 9,0 & 17,9 & 59,6 & 8,9 & 1,5 \\
\hline \multicolumn{8}{|l|}{ Cigarrillos } \\
\hline \multirow{2}{*}{ Alguna vez } & Público & 13,9 & 33,6 & 13,7 & 21,9 & 14,9 & 2,0 \\
\hline & Privado & 14,0 & 41,0 & 18,4 & 17,0 & 8,5 & 1,0 \\
\hline \multirow{2}{*}{ Frecuentemente } & Público & 4,9 & 9,2 & 14,4 & 57,4 & 11,1 & 2,9 \\
\hline & Privado & 2,8 & 7,7 & 17,8 & 65,1 & 5,7 & 1,4 \\
\hline \multicolumn{8}{|c|}{ Drogas ilegales } \\
\hline \multicolumn{8}{|l|}{ Marihuana } \\
\hline \multirow{2}{*}{ Alguna vez } & Público & 6,7 & 18,3 & 22,4 & 32,6 & 15,8 & 4,2 \\
\hline & Privado & 4,6 & 17,8 & 28,5 & 38,8 & 8,2 & 2,1 \\
\hline \multirow{2}{*}{ Frecuentemente } & Público & 4,5 & 5,1 & 8,9 & 61,1 & 16,0 & 4,4 \\
\hline & Privado & 2,1 & 3,6 & 9,0 & 74,1 & 8,9 & 2,2 \\
\hline \multicolumn{8}{|l|}{ Pbc o crack } \\
\hline \multirow{2}{*}{ Alguna vez } & Público & 5,3 & 13,5 & 21,7 & 35,3 & 19,4 & 4,8 \\
\hline & Privado & 3,3 & 13,0 & 26,1 & 43,8 & 11,4 & 2,5 \\
\hline \multirow{2}{*}{ Frecuentemente } & Público & 4,4 & 4,5 & 7,2 & 58,9 & 20,3 & 4,7 \\
\hline & Privado & 2,1 & 2,9 & 6,9 & 72,8 & 12,7 & 2,6 \\
\hline \multicolumn{8}{|l|}{ Cocaína } \\
\hline \multirow{2}{*}{ Alguna vez } & Público & 5,6 & 16,1 & 23,4 & 33,9 & 16,3 & 4,7 \\
\hline & Privado & 3,5 & 14,9 & 28,7 & 41,7 & 8,8 & 2,9 \\
\hline \multirow{2}{*}{ Frecuentemente } & Público & 4,5 & 4,8 & 8,3 & 60,8 & 17,1 & 4,5 \\
\hline & Privado & 2,2 & 3,4 & 7,7 & 74,5 & 10,0 & 2,4 \\
\hline \multicolumn{8}{|l|}{ Inhalantes } \\
\hline Alouna yez & Público & 5,0 & 8,9 & 14,5 & 49,9 & 18,1 & 3,6 \\
\hline & Privado & 2,9 & 8,9 & 19,7 & 55,9 & 10,8 & 1,7 \\
\hline Frecuentemente & Público & 5,0 & 6,6 & 9,7 & 55,6 & 19,7 & 3,5 \\
\hline Frecuentemente & Privado & 2,7 & 4,4 & 10,4 & 69,2 & 11,7 & 1,7 \\
\hline Éxtasis & & & & & & & \\
\hline Alouna vez & Público & 5,2 & 13,6 & 21,2 & 34,9 & 20,3 & 4,8 \\
\hline Aiguna vez & Privado & 3,4 & 12,6 & 26,0 & 43,5 & 12,0 & 2,6 \\
\hline & Público & 4,7 & 3,4 & 5,7 & 59,1 & 22,5 & 4,6 \\
\hline Frecuentemente & Privado & 2,4 & 2,1 & 5,1 & 73,7 & 14,1 & 2,5 \\
\hline & & & ss médic & & & & \\
\hline Tranquilizante & & & & & & & \\
\hline Alguna vez & Público & 6,5 & 14,1 & 17,5 & 41,6 & 17,1 & 3,2 \\
\hline Aigund vez & Privado & 4,8 & 17,0 & 25,1 & 40,7 & 10,7 & 1,6 \\
\hline Frecuentemente & Público & 5,0 & 7,2 & 11,0 & 55,2 & 18,1 & 3,4 \\
\hline Frecuentemente & Privado & 2,6 & 5,9 & 14,0 & 64,3 & 11,5 & 1,7 \\
\hline
\end{tabular}


consumo frecuente entre el 47,4 y 50,9\% de la población con el mismo perfil lo considera un «gran riesgo». En el caso de consumo de inhalantes el 42,2\% de la población escolar que vive con su pareja o conviviente considera de «gran riesgo» el aspirado de inhalantes de manera ocasional, mientras que $44,6 \%$ de escolares con el mismo perfil considera de «gran riesgo» el aspirado frecuente de inhalantes.

Son los escolares que viven con su pareja o conviviente los que presentan en menor porcentaje, comparado con los escolares de diferente perfil familiar, la percepción de «gran riesgo» el consumo ocasional de tranquilizantes sea cuando el consumo es ocasional o frecuente.

\section{DISCUSIÓN}

El consumo de drogas, así como la edad de inicio, han sufrido cambios alarmantes, producto de su mayor disponibilidad y acceso al alcance de niños y adolescentes (9). Las estadísticas muestran que 8 de cada 100 personas entre los 11 y 19 años admitieron haber consumido sustancias «alguna vez en su vida», empezando con el tabaco entre los 11 y 14 años y el consumo de alcohol entre 15 y 19 años, por lo que representan las tasas más altas de consumo, se argumenta como causa de consumo: la curiosidad, la influencia de los amigos, la moda, la depresión, o la falta de comunicación en la familia (9). La búsqueda de sensaciones y la percepción de invulnerabilidad hacia el peligro son los principales predictores de comportamientos riesgosos para la salud adolescente, entre los cuales se encuentra predominantemente el uso de drogas (1011).

En la investigación se encontró que las mujeres escolares (tabla 1) percibieron el consumo de drogas ilegales ocasional o frecuentemente en «gran riesgo», manifestando una actitud desfavorable hacia estas sustancias. Los escolares tienden a interpretar la información respecto a los efectos negativos del consumo de sustancias adictivas en función de su nivel de conocimiento; es decir, buscan congruencia entre lo que interpretan de la realidad, su conducta y hábitos cotidianos sobre los efectos negativos del consumo de sustancias adictivas (legales, ilegales y médicas) (12). Es necesario desmontar determinadas creencias (muy arraigadas en la cultura popular) en torno a la manera en la que el alcohol, y las drogas afectan al comportamiento humano, sobre todo en los hombres, ya que esta vinculación se da a que el hombre es un ente social, lo cual es normal que esté expuesto a las drogas, e incluso que sea consumidor, por lo que se interpreta como una actividad propia de su sexo (13). Situación diferente en- contró Gil, quien encontró que las mujeres que consumen cierto tipo de drogas, se les considera como una desviación de lo que la sociedad entiende como «concepto de ser mujer», o de lo que se espera de ella (14).

En la evaluación por edades (tabla 2) se encontró que a medida que los escolares crecen y avanzan en su educación su percepción a «gran riesgo» también aumenta de manera significativa (con todos los tipos de drogas). Una de las teorías más utilizadas para poder educar a la población escolar de edades y grados inferiores es mediante programas y campañas de promoción y prevención de salud con estrategias de recurso al miedo. La teoría de la acción motivadora protectora afirma que podemos aumentar significativamente la intención de mantener una conducta de salud si conseguimos infundir el suficiente miedo en el sujeto, siempre y cuando se le dé también salidas posibles y eficaces para evitar el consumo de drogas. Una vez que el sujeto está motivado hacia la protección los comportamientos que lleve a cabo irán en consonancia con la evitación del riesgo (7). Se sabe que dentro del contexto escolar se persigue el cultivo y la promoción de dimensiones que se relacionan con la aparición o la ausencia de los factores que están relacionadas a la base de las conductas. Se aborda el fomento de aquellas condiciones que favorecen al adolescente en su progresiva maduración y autonomía (13). Este autor explica que si la necesidad apuntada por los jóvenes para iniciarse en el consumo de drogas es la de experimentar sensaciones nuevas, adaptarse al mundo social, combatir el aburrimiento, manejar el tiempo de ocio, etc., la escuela, en ese sentido, ofrece un marco ventajoso donde cabe posibilitar alternativas para que el adolescente pueda satisfacer su curiosidad y su necesidad de nuevas experiencias enseñándole a planificar su ocio y facilitándole el contacto con sus iguales, todo ello desde un punto de vista constructivo. Puesto que el consumo de drogas también ayudaría a no aburrirse en fiestas y sentirse bien (15).

$\mathrm{Al}$ analizar la situación de la percepción de riesgo en Lima, Callao y provincias (tabla 3), se pudo determinar que los estudiantes de Lima (72\%) presentan una mayor percepción a «gran riesgo» sobre el consumo de drogas ilegales, seguido por Callao $(68,2 \%)$ y finalmente las provincias $(62,2 \%)$ del país, aunque es cierto que es más de la mitad de la población que reconoce la peligrosidad del consumo de drogas, se debe de dar una mayor capacitación y enfocarse en la labor preventiva para que esta logre captar un 100\% en la población escolar, y poder así disminuir posibles consumidores. En lo que a las drogas legales e ilegales se refiere, las aportaciones etnográficas han demostrado que su consumo no tiene ni el mismo significado ni 
el mismo efecto en todas las culturas, épocas o contextos sociales, ya que son muy diversos (16).

En la tabla 4 se observa que el mayor porcentaje de percepción «a gran riesgo» a las drogas ilegales se encuentra en las escuelas privadas $(74,5 \%)$ mientras que en los públicos este porcentaje es de 58,9\%, nos lleva a pensar que la educación sobre el consumo de drogas en estas escuelas son diferentes o la influencia del estatus social condiciona la percepción en los estudiantes, ya que se considera que los que cuentan con mayores recursos pueden pagar por una mejor educación y los que no solo van a escuelas públicas donde el entorno del escolar es muy diferente e ineficiente. Esta idea podría ser errónea ya que el consumo de drogas se da en ambos tipos de colegios, es cierto que siempre hay una mayor inclinación de consumo o de déficit de educación en la población escolar de entidades educativas públicas por lo cual debe existir capacitación o implementación de charlas y actividades dirigidas hacia el no consumo de drogas.

$\mathrm{Al}$ analizar la percepción de riesgo según el tipo de familia de los escolares (tabla 5), se determinó una similitud de porcentajes (51,4 y 61,4\%) a "gran riesgo» en el uso frecuente de drogas legales (alcohol y cigarrillos). Por otro lado, se evidencia que los escolares que viven con ambos padres son los que presentan una mayor percepción de riesgo al consumo de drogas ilícitas y médicas, tomando así importancia la constitución del hogar en el escolar y cómo este influye en sus decisiones. La familia cumple una función importante, ya que es un pilar fundamental en los adolescentes, no solo porque conforma el espacio de socialización por excelencia, sino porque es aquí donde los adolescentes buscan las pautas fundamentales para orientarse en la vida (14). Desde el nacimiento hasta la adolescencia la familia es un ámbito privilegiado de formación de actitudes, habilidades y valores que permitirán que, posteriormente, el sujeto afronte una etapa vital decisiva, como es la adolescencia, lo cual se evidencia en la tabla analizada, que todos los escolares que viven con sus padres, uno de ellos, o algún familiar que representa esa imagen familiar, tienen una mayor percepción de riesgo que los escolares que viven sus parejas (44,3\%).

Entonces, se podría entender que para aumentar el porcentaje de la percepción de riesgo en los escolares se debe brindar sesiones de prevención en las diferentes instituciones educativas y que, a su vez, estas deben ser reforzadas y actualizadas para poder mantener una percepción alta del riesgo entre los escolares para disminuir la probabilidades de consumo, esto también dependerá de quiénes dicten estas charlas preventivas, los cuales tienen que estar capacitados adecuadamente (17).

Para la práctica de enfermería (18) frente al consumo de drogas es importante la formación y experiencia, mientras que por los contextos en que se trabaja se pueden brindar cuidados en los hogares, escuelas, trabajo, prisiones, clínicas de salud y bienestar, y otros en la comunidad, así como en los hospitales y centros de investigación. En casi todos los países, las enfermeras son el grupo más numeroso de dispensadores de cuidados de salud. Las enfermeras son también de importancia esencial para la formación y la supervisión de otro personal y para la planificación, la organización, la vigilancia y la evaluación de los servicios brindados, en este particular para la prevención y control del consumo de drogas (18-19).

La prevención es el recurso más poderoso para enfrentar el consumo de drogas, cuya expansión mundial empezó hace 40 años. La prevención comienza en hogares estables, armoniosos, estableciendo estilos de vida alejados de las drogas, el desarrollo de valores morales, el culto a la espiritualidad y dignidad del hombre, la práctica de deporte para hacer realidad el principio de mente sana en cuerpo sano, la educación para la salud desde fases tempranas y el desarrollo de una actitud social de total intolerancia al consumo de sustancias (20). Los problemas generados por la dependencia y abuso de droga requieren un abordaje sistematizado, riguroso y eficaz de la enfermera como profesional de salud (21).

\section{REFERENCIAS BIBLIOGRÁFICAS}

1. DEVIDA. III Estudio Nacional de prevención y consumo de drogas en Estudiantes de Secundaria 2009: Resultados Nacionales Lima - Perú. 2009.

2. García del Castillo JA. Concepto de percepción de riesgo y su repercusión en las adicciones. Health and Addictions / Salud y Drogas. 2012. 12(2):138-140

3. Arrellanez HJL, Díaz NDB, Wagner EF, Pérez IV. Factores psicosociales asociados con el abuso y la dependencia de drogas entre adolescentes: Análisis bivariado de un estudio de casos y controles. Salud Mental. 2004. 27(3):54-65.

4. Salazar SF, Bustamante ChIV, Rodríguez SJE, Megías QI, Ballesteros GJC. Percepciones sociales sobre drogas en Lima (Perú). Lima: Fundación de Ayuda contra la Drogadicción (FAD). Comisión Nacional para el Desarrollo y Vida sin Drogas DEVIDA; 2011.

5. Organización de los Estados Americanos, Secretaria de Seguridad Multidimensional, Comisión Interamericana para el Control del Abuso de Drogas, 
Observatorio Interamericano de Drogas. Informe del Uso de Drogas en Las Américas OEA; 2011.

6. Ajzen I. From intentions to actions: A theory of planned behaviour. In: Kuhl J, Beckmann J. Action-control: From cognition to behaviour. Heidelberg: Springer; 1985. Pag.11-39.

7. Harris P, Middleton W. The illusion of control and optimism about health: on being less at risk but no more in control than others. Br J Soc Psychol. 1994: 33(Pt 4):369-86.

8. Medina AS, León RJM. Modelos explicativos de la psicología de la salud. En León J, Medina S, Barriga S, Ballesteros A, Herrera I (Eds.). Psicología de la salud y de la calidad de vida. Barcelona: Universidad Oberta de Catalunya; 2004.

9. Ortiz OG, Ramírez ChBR, Tapia PDC. Percepción de riesgo sobre las drogas de inicio por los adolescentes con nivel escolar de secundaria. 2008. Disponible en: http://es.scribd.com/doc/49325091/ PERCEPCION-DE-RIESGO-SOBRE-LASD R O G A S - D E - I N I C I O - P O R - L O S ADOLESCENTES-CON-NIVEL-ESCOLAR-DESECUNDARIA

10. Rebolledo EAO, de Medina NMO, Pillon, SC. Fatores de riscos associados ao uso de drogas entre estudantes adolescentes. Revista Latino-Americana de Enfermagem. 2004 12(spe), 369-375.

11. Ravert RD, Schwarts SJ, Zamboanga BL, Kim SY, Weisskirch RS, Bersamin M. Sensation seeking and danger invulnerability: Paths to college student risktaking. Personality and Individual Differences. 2009. 47(7), 763-8.

12. Uribe AJI, Verdugo LJC, Zacarías SX. Relación entre percepción de riesgo y consumo de drogas en estudiantes de bachillerato. Psicología y Salud. EneroJunio. 2011. 21(1):47-55.

13. Oñate P. Prevención educacional de las toxicomanías: Criterios básicos. Comunidad y Drogas. 1987. (3): 83-89.

14. Musayón OY, Torres DC, Sánchez DE, Chavéz CE. Factores de riesgo del consumo de bebidas alcohólicas en escolares de educación secundaria. Invest. educ. enferm. 2005. 23(1), 54-67.

15. Gil GE, Romo AN, Poo RM, Meneses FC, Markez AI, Vega FA. Género y psicofármacos: la opinión de los prescriptores a través de una investigación cualitativa. Atención Primaria. 2005. 35(8) 402-7.

16. Sierra D, Pérez $M$, Pérez A, Núñez $M$. Representaciones sociales en jóvenes consumidores y no consumidores de sustancias psicoactivas. Adicciones.2005. 17(4), 349-360.

17. Almanza SEE, Pillon SC. Programa para fortalecer fatores protetores que limitam o consumo de tabaco e álcool entre estudantes de educação média. Revista Latino-Americana de Enfermagem. 2004. 12(spe), 324-332.

18. CIE. La enfermería y la atención primaria de salud: Una fuerza unificada. Ginebra; 1998.

19. Wright MGM. Musayón OFY, Alayo SM. La Contribucción de las Escuelas de Enfermería en el Área de Reducción de la Demanda de las Drogas en América Latina. CICAD/SSM/OEA. Washington; 2012.

20. Sandoval JE. Una Estrategia de Capacitación en la Prevención del Alcoholismo. Rev. Hosp. Psiq. Hab. 1997. 38(1): 51-5.

21. Miotto WMG. «La contribución de la enfermería frente al fenómeno de las drogas y la violencia en América latina: un proceso de construcción.» Ciencia y enfermería. 2002. 8(2):09-19.

\section{Correspondencia}

Lorena Podestá Alegre

Universidad Peruana Cayetano Heredia

Dirección: Av. Honorio Delgado 430, Urb. Ingeniería, S.M.P.

Correo electrónico: lorena.podesta@upch.pe

Forma de citar este artículo: Ojeda- Oyague N, Podestá- Alegre L, Musayón-Oblitas F Y. Percepción de riesgo sobre el consumo de drogas en escolares de secundaria de Lima - Perú. Rev. enferm Herediana.2014;7(1):24-37. 